\title{
Transitions in Social Network Types over Time among Older Adults
}

\author{
Pildoo Sung $^{a}$ Rahul Malhotra ${ }^{a, b}$ Grand H.-L. Cheng ${ }^{c}$ \\ Angelique Wei-Ming Chan ${ }^{\mathrm{a}}$ b \\ ${ }^{a}$ Centre for Ageing Research and Education, Duke-NUS Medical School, Singapore, Singapore; \\ bHealth Services and Systems Research, Duke-NUS Medical School, Singapore, Singapore; \\ 'School of Arts and Social Sciences, The Open University of Hong Kong, Hong Kong, Hong Kong SAR
}

\section{Keywords}

Social network typology - Change in social network types . Latent transition analysis

\begin{abstract}
Objective: Network typology studies have identified heterogeneous types of older adults' social networks. However, little is known about stability and change in social network types over time. We investigate transitions in social network types among older adults, aged 60 years and older, and factors associated with such transitions. Methods: We used data on 1,305 older adults, participating in 2 waves of a national, longitudinal survey, conducted in 2016-2017 and 2019 , in Singapore. Latent transition analysis identified the distinct types of social networks and their transition patterns between the waves. Multinomial logistic regression examined the association of baseline and change in physical, functional, and mental health and baseline sociodemographic characteristics with network transitions into more diverse or less diverse types. Results: We found 5 social network types at both waves, representing the most to the least diverse types - diverse, unmarried and diverse, extended family, immediate family, and restricted. Between waves, about $57 \%$ of respondents retained their social network type, whereas $24 \%$ transitioned into more diverse types and $19 \%$ into less diverse types. Those who were older and less
\end{abstract}

educated and those with worsening functional and mental health were more likely to transition into less diverse types versus remaining in the same type. Discussion: The findings capture the dynamics in social network composition among older adults in the contemporary aging society. We highlight sociodemographic and health disparities contributing to later life social network diversity.

(c) 2022 The Author(s)

Published by S. Karger AG, Basel

\section{Introduction}

Social networks contribute to successful aging, which is often challenged by declining health and social withdrawals $[1,2]$. To capture benefits embedded in and arising from social networks in later life, considerable literature has focused on single specific attributes of social networks such as core network ties, employing a variablecentered approach $[3,4]$. A network typology analysis, on the other hand, adopts a person-centered approach to highlight multidimensional, interactive, and overlapping features of social networks among older adults [5]. Applying clustering or latent class analysis to a set of net-

Grand H.-L. Cheng changed his affiliation to Department of Medicine, Yong Loo Lin School of Medicine, National University of Singapore, Singapore, Singapore during the revision process.
C 2022 The Author(s).

Published by S. Karger AG, Basel

This is an Open Access article licensed under the Creative Commons Attribution-NonCommercial-4.0 International License (CC BY-NC) (http://www.karger.com/Services/OpenAccessLicense), applicable to the online version of the article only. Usage and distribution for commercial purposes requires written permission.
Correspondence to:

Pildoo Sung, pildoo.sung@ duke-nus.edu.sg 
work indicators, typology studies have classified older adults into distinct types of social networks, internally homogeneous and externally heterogeneous [6]. In doing so, relevant studies have unveiled the health and well-being disparities between social network types $[7,8]$.

In general, the literature has identified 4 common types of social networks: diverse and socially engaged, friendoriented, family-centered, and restricted [7, 8]. A diverse and socially engaged type characterizes older adults having diversified social ties with family, friends, neighbors, and acquaintances from community engagement. A friendoriented type indicates older adults' networks predominantly composed of friends. A family-focused type represents older adults whose social networks are made up primarily of immediate family members. A restricted type categorizes those who lack social connectedness in general. While informative, the relatively nascent studies on social network typology suggest contextual variation $[9,10]$. For instance, Litwin and Shiovitz-Ezra [11] found a congregate social network type among older Americans that comprises those who frequently attend religious services. Cheng et al. [12] showed the presence of a distant family type, characterized by high levels of contact and support exchange with distant kin, among Chinese older adults in Hong Kong. These studies call for the need to assess network typologies in different sociocultural contexts.

Moreover, most typology research have investigated types of older adults' social networks cross-sectionally, leaving their dynamic nature underexplored [6]. How likely are older adults to change their social network type over time? Who are more likely to transition into different social network types? Researchers have shown that the composition of older adults' social networks changes as they age $[3,13]$. Socioemotional selectivity theory proposes that people prioritize emotionally close ties as they age, due to the awareness of the limited time left in their life [14]. Likewise, the social convoy model suggests that older adults are likely to limit their attention to close family and friends, maintaining supportive convoys of social relations [15]. Older adults are thus expected to gradually lose diversity in their social networks while focusing on a few close relationships with family and a circle of intimates. A few typology studies, investigating changes in older adults' social network types, have supported the abovementioned theories on social network concentration $[7,8]$. These studies have shown that older adults tend to transition from more diverse into less diverse types over time [7, 8]. For instance, Li and Zhang [7] found that about $60 \%$ of Chinese older adults, categorized into the diverse type in 2005 , transitioned into the family $(30 \%)$ or restricted types (30\%) in 2012. Kim et al. [8] also showed that about $70 \%$ of older Koreans in the diverse type transitioned into less diverse types between 2006 and 2008.

Nevertheless, recent empirical evidence has reported a pattern of growth or stability in older adults' social networks $[3,4,13,16]$. Research on older Americans found that more than $80 \%$ of older adults add one or more new confidants to their social networks in 5 years [3]. Studies analyzing European older adults also reported a general trend of expansion in confidant networks in 4 years [4, 16]. Yet, these studies have mainly focused on cultivation of strong ties, measured by a name generator asking older adults with whom they discuss important matters [17]. It is thus uncertain whether increasing reliance on confidant networks is accompanied by a gradual loss of weak ties, such as those formed through attendance at religious services or voluntary organizations, engendering losing diversity in overall social network composition. Therefore, our study revisits changes in types of older adults' social networks using a person-centered approach.

Exploring transitions in social network types opens the possibility of investigating factors associated with changes in social network composition. Specifically, the health-begets-composition hypothesis suggests that health influences the composition of social networks among older adults [18]. For instance, it is plausible that older adults who suffer from multimorbidity are more likely to form social networks mainly composed of family members for caregiving, while gradually losing ties beyond their kin boundaries. Empirically, Li and Zhang [7] showed that poorer health at baseline predicted a higher relative risk of having family-focused or restricted network types than a diverse type at follow-up, although the association between change in health and transitions in network types was not explicitly modeled. More empirical evidence is needed to confirm the presence and pattern of association between declining health and change in social network types.

Additionally, studies have reported sociodemographic disparities in the composition of social networks among older adults $[6,8]$. For instance, age is known to be negatively related to network diversity due to reduced social demands, coupled with an individual's preference for emotionally close ties in later life [2, 19]. Females are more likely to have diversified social networks than males, while ethnic minorities may have less diverse social networks [20]. Socioeconomic status (SES), defined by household income or education, determines opportunities and capacities for the maintenance and utilization of nonkin ties among older adults $[19,21]$. Less is known 
however about whether these sociodemographic characteristics are associated with changes in social network types over time.

To fill these gaps, our study investigates (1) the distinct types of social networks in Singapore, (2) transitions in social network types over time, and (3) the association of change in health and sociodemographic characteristics with transitions to more diverse or less diverse network types. In doing so, we add to the relevant literature in 3 ways. First, we provide the first systematic examination of the social network typology among older adults in Singapore, a rapidly aging Asian nation projected to become the fifth oldest country in the world by 2050 [22]. Second, we examine transitions in social network types using latent transition analysis (LTA), a person-centered analytical approach that rigorously captures the dynamics in social network types $[23,24]$. Third, we identify the core factors associated with changes in social network composition in later life.

Building upon the literature [12], we expect to find older Singaporeans who predominantly interact with their extended family, in addition to other common social network types (hypothesis 1). This is mainly due to the prevalence and variations in family-based network types in collectivistic Asian societies where the extended family plays a key role in providing instrumental and emotional support $[9,12]$. We further posit that older Singaporeans are more likely to lose diversity in their social networks by transitioning from more diverse into less diverse social network types than the other way around (hypothesis 2). This is based on theories projecting network concentration $[14,15]$ and empirical findings showing reduced diversity in older adults' social network composition over time $[7,8,25]$. We assume that losing diversity in social network composition is compatible with maintaining or gaining close ties with family and friends over time [3]. Last, we expect that older adults with deteriorating health and low SES are likely to experience withdrawal from more diverse to less diverse network types due to their lack of capabilities and resources in maintaining diversity in their social networks as they age (hypothesis 3).

\section{Materials and Methods}

Dataset and Analysis Sample

We used data from the 2 waves of the Transitions in Health, Employment, Social engagement and Inter-Generational transfers in Singapore Study (THE SIGNS study), a nationally representative longitudinal survey of community-dwelling older Singapore citizens and permanent residents aged 60 years and above. Wave 1 was conducted in 2016-2017 and wave 2 in 2019. Stratified ran- dom sampling, based on age, gender, and ethnicity from the 2015 population estimates, was used to select wave 1 participants. Those aged 75 years or above and of Malay and Indian ethnicity were oversampled. Data collection was through face-to-face interviews, after informed consent, in both waves.

A total of 4,549 older adults were recruited in wave 1 with a response rate of $50.7 \%$. At wave 1, respondents were randomly allocated, within each stratum defined by age, gender, and ethnicity, 1 out of 2 questionnaire versions. Sociodemographic characteristics and health status (based on questions common to both questionnaire versions) of participants who responded to the 2 versions were nearly identical. Questions about some of the social network variables used in this article - community participation, religious service attendance, and depressive symptoms - were unique to a version. Thus, 2,277 out of the 4,549 respondents, who were not asked these questions, were excluded from the analysis, leaving 2,272 respondents [26]. Of the 2,272 respondents, 1,443 were reinterviewed in wave 2 (retention rate $=64 \%$ ). While those of higher age were more likely not to be reinterviewed, the retention rate is comparable to other national surveys conducted earlier in Singapore [27]. After excluding proxy respondents (who answered on behalf of the older adult unable to respond himself or herself due to health reasons) who were not asked some of the social network variables, our final analysis sample comprised 1,305 respondents who answered at least one or more questions on their social network at both waves (see online suppl. Fig. 1 for the flow chart; see www.karger.com/doi/10.1159/000521213 for all online suppl. material).

It is plausible that the exclusion of survey participants from our analysis sample, due to random allocation of questionnaire versions at wave 1 and attrition between waves, affected our results. We thus conducted sensitivity analyses using multiple imputations (MIs), assuming that the missing information can be inferred from the observed characteristics of the participants [28] (i.e., missing at random assumption) (See online suppl. Text 1 for more details). Specifically, we replicated the analysis described below in $3 \mathrm{im}$ puted data sets: (1) around $50 \%$ of the values, which were missing due to random allocation of questionnaire versions, were imputed; (2) around $40 \%$ of the values, which were missing due to sample attrition, were imputed; and (3) around $70 \%$ of the values imputed, which were missing due to random allocation of questionnaire versions and sample attrition, were imputed.

\section{Measures}

Social Network Indicators

We used 9 social network variables to derive social network indicators, which were then used in LTA to examine social network types and their transitions. Variables were dichotomized to facilitate model identification and result interpretation, which is common in the literature using LCA and LTA $[26,29,30]$. Our indicator-specific dichotomization procedure carefully considered the meaning and distribution of variables while securing meaningful variations in conducting LTA [31].

Of the 9 variables, the first 3 were related to immediate family ties: (1) living arrangement was recorded as living alone or living with others; (2) marital status as married or nonmarried (widowed, separated, divorced, and never married); and (3) the number of children as having at least 1 living child or no living child. Three network indicators were thus derived: (1) live alone, (2) married, and (3) have one or more living children. 
The next 4 variables were adopted from the Lubben social network scale, measuring social networks with relatives and friends outside the household [32]. Variables (4) and (5), asking the number of relatives and friends, respectively, one sees or hears from at least once a month, had 6 response options: $0,1,2,3-4,5-8$, and $>9$. We combined the first 3 response options into one category $(0,1$, and 2$)$ and the remaining 3 response options into another category ( 3 or above), considering their meaning and distribution (online suppl. Table 1) $[29,33]$. Variables (6) and (7), measuring the contact frequency with the closest relatives and friends, respectively, also had 6 response options: never, seldom, sometimes, often, very often, and always. Similarly, we combined never, seldom, and sometimes into one category and often, very often, and always into another to identify older adults who had frequent contact with their closest relatives/friends, taking into account the meaning and distribution of the responses and largely corresponding to the previous studies (e.g., [31]). In short, 4 dichotomous indicators were constructed from the Lubben social network scale: (4) have 3 or more relatives to contact, (5) have 3 or more friends to contact, (6) have frequent contact with relatives, and (7) have frequent contact with friends.

The last 2 indicators assessed social networks arising from community engagement. Variables (8) attendance in community organizations and (9) attendance in religious services had 5 response categories: everyday/more than once a week, once a week, 2 or 3 times a month, once or more times a year, and not at all. Variable (8) was dichotomized as not at all (about $80 \%$ at wave 1) versus others, due to the highly skewed distribution (online suppl. Table 1). Regarding variable (9), the literature has usually considered those who attend religious services once a week or more often as frequent attendees [34]. We thus set once a week as a cut point (everyday/more than once a week/once a week vs. the rest). Two indicators were thus created: (8) ever attend community events and (9) attend religious services weekly or more.

\section{Baseline and Change in Physical, Functional, and Mental}

Health

We considered baseline and change in the 3 health domains. Number of chronic diseases was a sum of chronic physical ailments such as cancer, hypertension, and osteoporosis that respondents have ever been diagnosed with by medical professionals. The number of functional difficulties was a sum of reported difficulties in activities of daily living (6 items) such as eating and getting dressed and instrumental activities of daily living (7 items) including preparing meals and shopping for necessities at wave 1 (Cronbach's $\alpha=$ 0.87). Depressive symptoms were assessed by the 11 -item CES-D scale (e.g., "I felt sad" and "I felt depressed") at wave 1 with 3 response categories (none/rarely, sometimes, and often) for each item [35]. We summed the 11 items to produce a single measure (Cronbach's $\alpha=0.83$ ), ranging from 0 to 22 . We then constructed 3 change variables - change in the number of chronic diseases, change in the number of functional difficulties, and change in depressive symptoms - by subtracting wave 1 scores from wave 2 scores. A positive value of the change variables represented worse physical, functional, and mental health at wave 2 than wave 1 , respectively, whereas a negative value indicated improvement over time.

\section{Sociodemographic Characteristics}

We accounted for key sociodemographic characteristics known to be associated with older adults' social networks [2]. These included age (ranges from 60 to 91 years), female gender, minority ethnicity (Malay, Indian, or Others vs. Chinese), highest completed education (no formal education, primary school, secondary, postsecondary, and tertiary), working (working full or part time, not working, and never worked), and small housing (a proxy for SES: living in 1-2 room government-built flat; and the rest).

\section{Methods}

We applied LTA to examine transition in network types and multinomial logistic regression to assess the association of change in health and sociodemographic characteristics with the transition. LTA, a longitudinal extension of LCA, estimates stability and changes among unobserved subgroups (latent classes) within a population over time [24]. The initial stage of LTA consists of the measurement model in which multiple LCAs at different time points capture the underlying grouping of respondents using a set of observed indicators [36]. Then, the structural model of LTA specifies transition probabilities of latent groups using autoregressive modeling [36]. In doing so, the conditional probabilities of a respondent being in a certain group at time point $t+1$ are estimated, given that the respondent was in a specific group at the previous time point $t$.

Our analysis consisted of a set of sequential procedures [30,37]. First, we used LCA to explore the optimal number of network types at waves 1 and 2, based on theoretical validity and various fit indices [23]. Specifically, information criteria (Akaike information criterion [AIC], Bayesian information criterion [BIC], and sample size-adjusted BIC [SABIC]) assessed the relative model fit: the lower the value, the better the fit of the model to the data. Two likelihood tests, Lo-Mendell-Rubin adjusted likelihood ratio test (LMR) and Bootstrapped likelihood ratio test (BLRT), compared the neighboring models: if the test is significant $(p<0.05)$, the model with $\mathrm{k}$ class fits the data better than the model with $\mathrm{k}-1$ class [38]. The entropy index evaluated how different the identified groups are from one another: a value greater than 0.80 indicates a precise class distinction. The smallest class is recommended to have at least $5 \%$ of the total sample for reliability.

We then tested measurement invariance to determine whether to constrain measurement parameters, the conditional item response probabilities for latent classes, between waves [30]. Measurement invariance assumes that there is no difference in the way latent classes are constructed at different time points when the same number and type of classes are identified over time. Therefore, measurement invariance, if achieved, allows transitions solely to be attributed to the changes in latent classes rather than their composition $[23,24]$. We used information criteria (AIC/BIC/ SABIC) and the Satorra-Bentler scaled $\chi^{2}$ difference test to compare invariant and noninvariant models [39].

After that, the structural model was fitted using prefixed measurement parameters from the measurement model. This was to ensure independence between measurement and structural models [37]. Last, the likelihood of a respondent in a certain network type at baseline to transition into other types at follow-up was estimated, after we assigned each older adult to the most likely network type at each wave based on the highest posterior probability. The robust maximum likelihood estimator was applied to the LTA model to handle missing values (around 2\%) in the observed network indicators.

Once the transitions were estimated, the association of the baseline sociodemographic characteristics and baseline and 
Table 1. Model fit indices for latent classes $(N=1,305)$

\begin{tabular}{|c|c|c|c|c|c|}
\hline & 3 class & 4 class & 5 class & 6 class & 7 class \\
\hline \multicolumn{6}{|l|}{ Wave 1} \\
\hline AIC & $11,812.16$ & $11,667.96$ & $11,580.54$ & $11,529.46$ & $11,512.09$ \\
\hline $\mathrm{BIC}$ & $11,962.21$ & $11,869.74$ & $11,834.06$ & $11,834.73$ & $11,869.09$ \\
\hline SABIC & $11,870.09$ & $11,745.86$ & $11,678.41$ & $11,647.31$ & $11,649.91$ \\
\hline$p(\mathrm{LMR})^{\mathrm{a}}$ & $<0.001$ & $<0.001$ & $<0.001$ & $<0.001$ & 0.18 \\
\hline$p(\mathrm{BLRT})^{\mathrm{b}}$ & $<0.001$ & $<0.001$ & $<0.001$ & $<0.001$ & $<0.001$ \\
\hline Entropy & 0.73 & 0.77 & 0.84 & 0.83 & 0.84 \\
\hline Smallest class, $n(\%)$ & $209(16)$ & $95(7)$ & $78(6)$ & $78(6)$ & $22(2)$ \\
\hline \multicolumn{6}{|l|}{ Wave 2} \\
\hline AIC & $11,849.63$ & $11,745.33$ & $11,662.44$ & $11,636.60$ & $11,613.26$ \\
\hline $\mathrm{BIC}$ & $11,999.67$ & $11,947.12$ & $11,915.96$ & $11,941.86$ & $11,970.26$ \\
\hline SABIC & $11,907.55$ & $11,823.23$ & $11,760.31$ & $11,754.45$ & $11,751.08$ \\
\hline$p(\mathrm{LMR})$ & $<0.001$ & 0.001 & $<0.001$ & 0.12 & 0.19 \\
\hline$p$ (BLRT) & $<0.001$ & $<0.001$ & $<0.001$ & $<0.001$ & $<0.001$ \\
\hline Entropy & 0.74 & 0.81 & 0.85 & 0.81 & 0.79 \\
\hline Smallest class, $n(\%)$ & $223(17)$ & $81(6)$ & $72(6)$ & $61(5)$ & $54(4)$ \\
\hline
\end{tabular}

AIC, Akaike information criterion. BIC, Bayesian information criterion. SABIC, Sample size adjusted baysian information criterion. LMR, Lo-Mendell-Rubin adjusted likelihood ratio test. BLRT, Bootstrapped likelihood ratio test. ${ }^{\mathrm{a}} p$ value from $\mathrm{LMR}$. ${ }^{\mathrm{b}} p$ value from BLRT.

change in health with transitions to more or to less diverse types, compared to those who remain stable, was assessed using multivariable multinomial logistic regression. Around $10 \%$ of the respondents did not respond to the questions or scales assessing functional difficulties and depressive symptoms. Thus, MIs by the chained equation were employed to handle the missing data. We used Mplus 8.5 for the LTA and Stata 16.1 for the regression.

\section{Results}

\section{Model Selection}

Table 1 reports the model fit indices of LCA models from 3 to 7 classes for wave 1 and 2 . We found that the 5 class model (representing 5 social network types) fitted the data best for both waves, with the lowest BIC (more parsimonious), the highest entropy (more precise classification), and a significant Lo-Mendell-Rubin LRT test (k class better than k-1 class) [23].

Since the same number and type of latent classes were derived at each considered time point, we tested for measurement invariance. The model fit comparison yielded conflicting results: while BIC and SABIC suggested the invariant model, the $\chi^{2}$ test supported the noninvariant model with $\chi^{2} \operatorname{diff}(45)=153.67, p<0.001$ [39] (see online suppl. Table 2). However, the $\chi^{2}$ test tends to be sensitive and unreliable when a large number of parameters are constrained to be equal [40]. Also, the literature recommends assuming measurement invariance when model fit indices conflict $[24,41]$ because measurement invariance facilitates model identification and estimation and clear interpretation of the transition probabilities [23]. We thus reasonably assumed measurement invariance of the model and fixed the item response probabilities to be equal at each wave for further analyses.

\section{Profiles of Social Network Types}

Figure 1 illustrates the item response probabilities of 5 distinct social network types, fixed at both waves, labeled as (1) diverse, (2) unmarried and diverse, (3) extended family, (4) immediate family, and (5) restricted, from the most diverse to the least. Numerical details are shown in online supplementary Table 3.

The diverse type comprised older adults having diversified social networks. These older adults were married, had children, and lived together with others. They tended to have 3 or more relatives and friends and had frequent contact with them. They were also relatively more likely to participate in community activities and religious services than those in other types.

The unmarried and diverse type shared similar characteristics to the diverse type except for living arrangements and marital status. While maintaining diverse networks outside their household, older adults with this type 


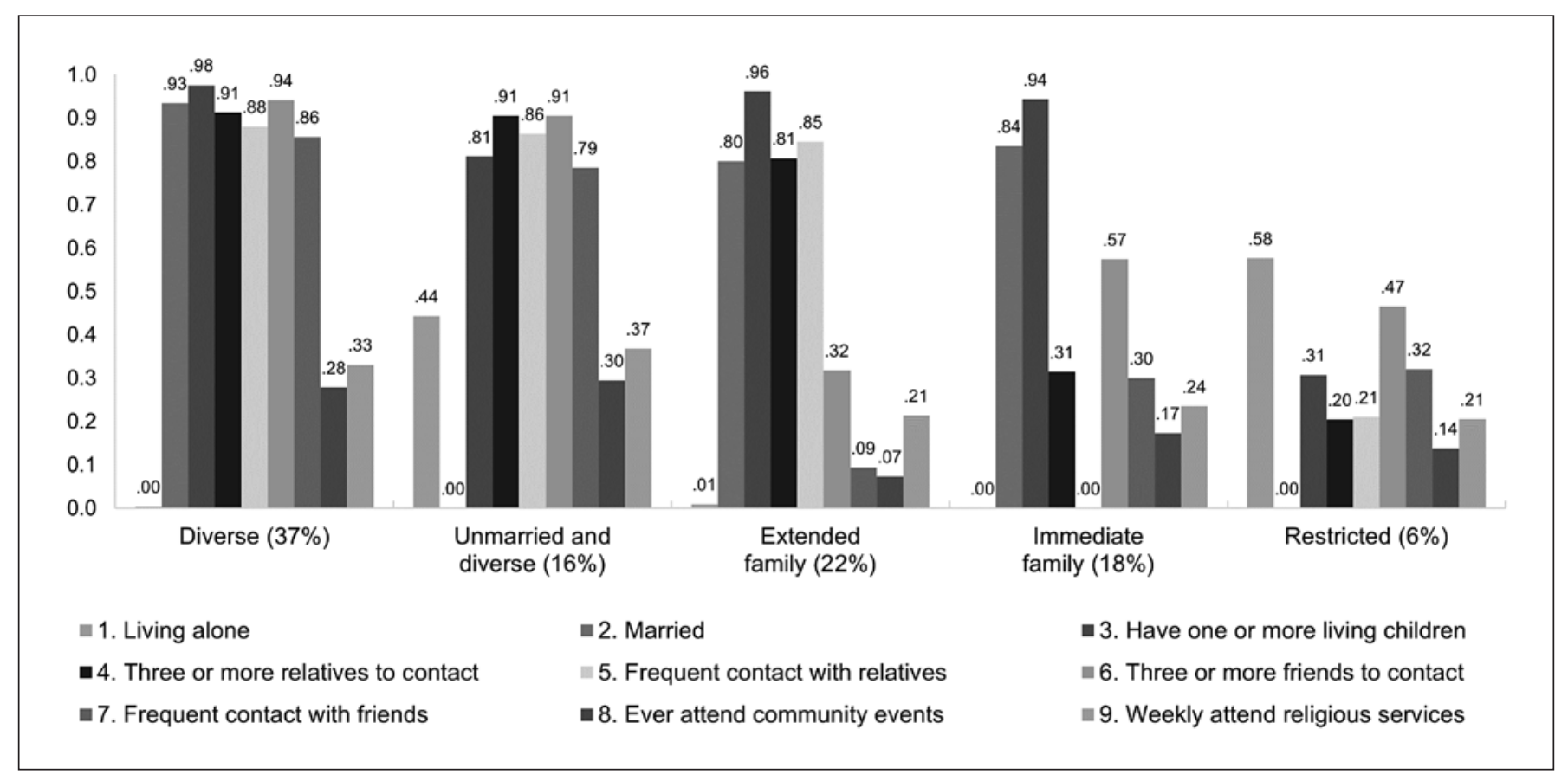

Fig. 1. Item response probabilities of 5 social network types. Proportions of network types in parenthesis were based on wave 1 data.

Table 2. Distribution of sociodemographic characteristics and health status by social network types based on the most likely network type pattern at wave 1

\begin{tabular}{|c|c|c|c|c|c|c|c|}
\hline \multirow[t]{3}{*}{ Characteristics, at wave 1 (range) } & \multicolumn{7}{|c|}{ Network type at wave 1} \\
\hline & total & diverse $[1]^{\mathrm{a}}$ & $\begin{array}{l}\text { unmarried and } \\
\text { diverse [2] }\end{array}$ & $\begin{array}{l}\text { extended } \\
\text { family [3] }\end{array}$ & $\begin{array}{l}\text { immediate } \\
\text { family [4] }\end{array}$ & $\begin{array}{l}\text { restricted } \\
{[5]}\end{array}$ & test statistics \\
\hline & $N(\%)$ & $485(37)$ & $209(16)$ & $290(22)$ & $241(18)$ & $80(6)$ & \\
\hline & \multicolumn{7}{|c|}{ Mean/column \% } \\
\hline \multicolumn{8}{|l|}{ Sociodemographic characteristics } \\
\hline Age (60-91), years & 70.63 & $69.73[2]$ & $72.89[1,3,4]$ & $70.84[2]$ & $70.05[2]$ & 71.24 & $F=1.02$ \\
\hline Female (female = 1), \% & 53 & 48 & 80 & 52 & 37 & 58 & $x^{2}=89.47^{* * *}$ \\
\hline Minority (non-Chinese $=1$ ), $\%$ & 24 & 25 & 26 & 25 & 23 & 19 & $x^{2}=1.77$ \\
\hline Education (0-3) & 1.34 & $1.46[2,3]$ & $1.19[1,4]$ & $1.18[1,4]$ & $1.45[2,3]$ & 1.29 & $F=1.39$ \\
\hline Working (working full/part time $=1$ ), $\%$ & 38 & 42 & 28 & 33 & 45 & 35 & $x^{2}=20.91^{* * *}$ \\
\hline Small housing $(1-2$ room housing $=1), \%$ & 9 & 5 & 13 & 5 & 10 & 28 & $x^{2}=58.13^{* * *}$ \\
\hline \multicolumn{8}{|l|}{ Health status } \\
\hline Chronic diseases (0-20), $n$ & 2.06 & 2.02 & 2.25 & 2.03 & 2.01 & 1.99 & $F=0.81$ \\
\hline Functional difficulties (0-13), $n$ & 0.27 & 0.16 & 0.25 & 0.36 & 0.33 & 0.40 & $F=1.06$ \\
\hline Depressive symptoms (0-22) & 2.81 & $2.30[3,4,5]$ & $2.64[5]$ & $2.99[1,5]$ & $3.21[1,5]$ & $4.60[1,2,3,4]$ & $F=3.92^{* * *}$ \\
\hline
\end{tabular}

Listwise deletion applied. ${ }^{* *} p<0.001$. ${ }^{*}$ Numbers in brackets represent statistically different group means from the Tukey comparison at $p<0.05$.

of social network were unmarried and more likely to live alone than their counterparts in the diverse type.

The extended family and immediate family types were 2 family-oriented social network types that differed in terms of extended family networks: those in the former type had 3 or more relatives to contact and had frequent interaction with them, while those in the latter type lacked. Otherwise, older adults classified in these 2 social network types were married, had children, and lived together with others; however, they fell short of friends' ties and community participation. 


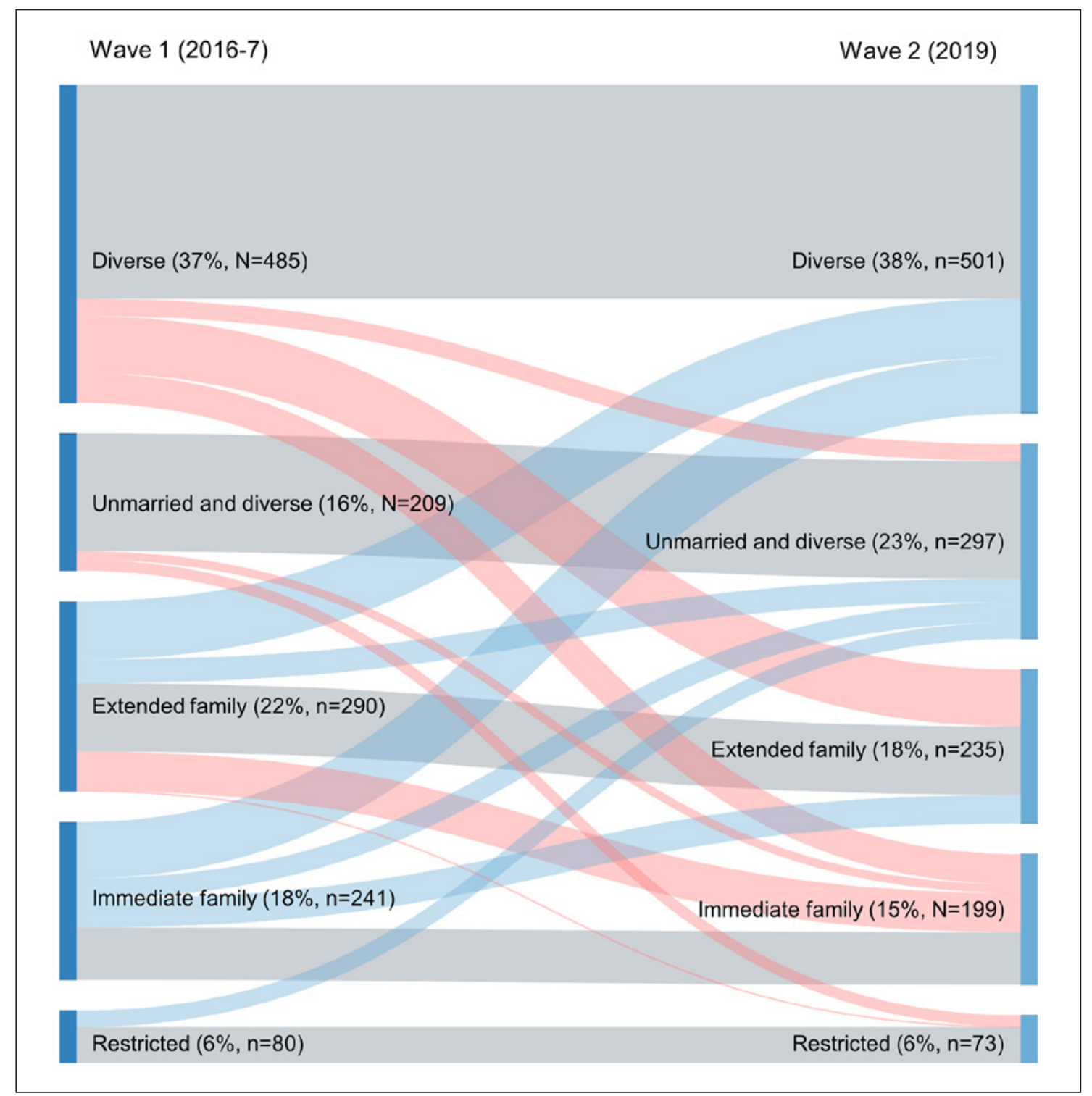

Fig. 2. Social network types and their transitions between the 2 waves based on the most likely network type pattern at each wave. Flows in blue represent transitions to more diverse type; flows in red denote transitions to less diverse type.

Older adults in the restricted type were unmarried, more than half of them lived alone, and two-thirds had no children. They were less likely to interact with relatives and friends or have community participation.

Table 2 provides the distribution of wave 1 sociodemographic and health variables by wave 1 network types. Notably, the unmarried and diverse type had the highest proportion of females and the lowest proportion of working respondents. Compared to other types, older adults in the restricted type were more likely to live in a small house and have a greater extent of depressive symptoms.

Transitions in Social Network Types
Transitions in Network Types between Waves

Figure 2 illustrates the prevalence of 5 network types at each wave, along with directed flows with a width proportional to the number of respondents who either remained in the same type at the 2 waves or transitioned from wave 1 types (left) into wave 2 types (right). Table 3 shows numerical details of transitions depicted in the figure.

At both waves, the most common social network type was the diverse type and the least was the restricted type. Between waves, $57 \%(n=744)$ of the respondents re- 
Table 3. Class count and proportions of network transitions between waves based on the most likely network type pattern

\begin{tabular}{|c|c|c|c|c|c|c|}
\hline & total & diverse & $\begin{array}{l}\text { unmarried and } \\
\text { diverse }\end{array}$ & $\begin{array}{l}\text { extended } \\
\text { family }\end{array}$ & immediate & restricted \\
\hline \multicolumn{7}{|l|}{ Network type at wave 1, $n(\%)$} \\
\hline Diverse & $485(37)$ & $326(67)$ & $26(5)$ & $87(18)$ & $46(9)$ & $0(0)$ \\
\hline Immediate family & $241(18)$ & $86(36)$ & $31(13)$ & $44(18)$ & $80(33)$ & $0(0)$ \\
\hline Restricted & $80(6)$ & $0(0)$ & $25(31)$ & $0(0)$ & $0(0)$ & $55(69)$ \\
\hline
\end{tabular}

Row percentage reported.

Table 4. Multinomial regression for the association of baseline and change in health status, and sociodemographic characteristics with transitions in network types $(N=1,305)$

\begin{tabular}{|c|c|c|c|c|}
\hline & \multicolumn{2}{|l|}{ Model 1} & \multicolumn{2}{|c|}{ Model 2} \\
\hline & \multicolumn{2}{|c|}{$\begin{array}{l}\text { network contraction: } \\
\text { transition to less diverse } \\
\text { types (ref: no transition) }\end{array}$} & \multicolumn{2}{|c|}{$\begin{array}{l}\text { network expansion: } \\
\text { transition to more diverse } \\
\text { types (ref: no transition) }\end{array}$} \\
\hline & $\mathrm{RR}$ & $95 \% \mathrm{Cl}$ & $\mathrm{RR}$ & $95 \% \mathrm{Cl}$ \\
\hline \multicolumn{5}{|l|}{ Change in health from wave 1 to wave 2} \\
\hline Change in the number of chronic diseases & 0.89 & $(0.76,1.03)$ & 0.99 & $(0.87,1.14)$ \\
\hline Change in the number of functional difficulties & $1.14^{*}$ & $(1.01,1.30)$ & 1.05 & $(0.93,1.19)$ \\
\hline Change in depressive symptoms & $1.09^{* * *}$ & $(1.04,1.15)$ & 0.96 & $(0.90,1.02)$ \\
\hline \multicolumn{5}{|l|}{ Health status at wave 1} \\
\hline Chronic conditions & 1.00 & $(0.90,1.11)$ & 1.05 & $(0.96,1.15)$ \\
\hline Functional difficulties & 0.91 & $(0.79,1.05)$ & 1.06 & $(0.94,1.19)$ \\
\hline Depressive symptoms & $1.07^{*}$ & $(1.01,1.14)$ & 1.01 & $(0.95,1.07)$ \\
\hline \multicolumn{5}{|l|}{ Sociodemographic characteristics at wave 1} \\
\hline Age & $1.03^{*}$ & $(1.01,1.05)$ & 1.01 & $(0.99,1.03)$ \\
\hline Female & $0.47^{* * *}$ & $(0.34,0.64)$ & $0.67^{* *}$ & $(0.51,0.89)$ \\
\hline Minority & 1.23 & $(0.87,1.73)$ & 1.25 & $(0.91,1.72)$ \\
\hline Education & $0.85^{*}$ & $(0.72,1.00)$ & 0.97 & $(0.84,1.11)$ \\
\hline Working & 1.39 & $(0.98,1.97)$ & 1.32 & $(0.98,1.80)$ \\
\hline Small housing & 0.64 & $(0.35,1.15)$ & 0.77 & $(0.47,1.25)$ \\
\hline
\end{tabular}

RR, Risk Ratio. Cl, Confidence Interval. Results are based on 20 imputed data sets. ${ }^{*} p<0.05$. ${ }^{* *} p<0.01 .{ }^{* *} p<$ 0.001

mained in the same type (flows in gray), while $43 \%$ ( $n=$ 561) transitioned into a different type. The most stable (over time) network type was unmarried and diverse more than four-fifths (86\%) of those in this type at wave 1 remained in it at wave 2 . Around $70 \%$ of respondents categorized in the diverse and the restricted types at wave 1 retained their network type at wave 2 .

Among 43\% who experienced a transition, we distinguished 2 categories of transitions. The first category rep- resented contraction, with respondents transitioning from more diverse into less diverse types (flows in red, $19 \%$, $[n=250])$. A majority of these older adults transitioned from diverse into extended family $(7 \%, n=87)$, extended into immediate family $(5 \%, n=60)$, diverse into immediate family $(4 \%, n=46)$, and diverse into unmarried and diverse $(2 \%, n=26)$ types.

The second category denoted expansion, with respondents moving from less diverse to more diverse types (flows 
in blue, $24 \%[n=311])$. These older adults included those transitioning from extended family into diverse $(7 \%, n=$ $89)$, immediate family into diverse $(7 \%, n=86)$, immediate family into extended family $(3 \%, n=44)$, extended family into unmarried and diverse $(3 \%, n=36)$, immediate family into unmarried and diverse $(2 \%, n=31)$, and restricted into unmarried and diverse $(2 \%, n=25)$ types.

\section{Factors Associated with Social Network Type}

\section{Transitions}

Table 4 reports findings from multinomial regressions for the association of baseline and change in health status and sociodemographic characteristics with transitions to less diverse types (network contraction) or more diverse types (network expansion), compared to retaining the same network types. Model 1 shows that age, baseline depressive symptoms, and change in the number of functional difficulties and depressive symptoms were positively associated with network contraction, whereas being female and higher levels of education were negatively related to such contraction. In other words, compared to those who remained in the same type, respondents had an increased risk of transitioning into less diverse social network types if they (1) were older (risk ratio $[\mathrm{RR}]=1.03$, confidence interval $[\mathrm{CI}]$ $=1.01-1.05)(2)$ reported a higher level of depressive symptoms at wave $1(\mathrm{RR}=1.07, \mathrm{CI}=1.01-1.14),(3)$ had more functional difficulties $(\mathrm{RR}=1.14, \mathrm{CI}=1.01-1.30)$, and (4) a greater extent of depressive symptoms $(\mathrm{RR}=1.09, \mathrm{CI}=$ $1.04-1.15$ ) at wave 2 versus wave 1 . In contrast, females ( $R R$ $=0.47, \mathrm{CI}=0.34-0.64)$ and those with higher levels of education $(\mathrm{RR}=0.85, \mathrm{CI}=0.72-1.00)$ had a lower risk of transitioning into less diverse types.

In model 2, no other variables except gender were associated with network expansion: females were less likely to experience transitions to more diverse types, compared to remaining in the same types $(\mathrm{RR}=0.67, \mathrm{CI}=0.51-$ $0.89)$. Overall, females tended to retain their social network type rather than gain or lose network diversity over time. Results from the 3 sensitivity analysis, presented in online supplementary Tables $5 \mathrm{a} / 5 \mathrm{~b}, 6 \mathrm{a} / 6 \mathrm{~b}$, and $7 \mathrm{a} / 7 \mathrm{~b}$, conducted after imputing missing values, confirmed that our main findings were robust.

\section{Discussion/Conclusion}

There has been growing scholarly interest in social network types among older adults in different sociocultural contexts (e.g., [9]). More importantly, only a handful of studies have examined transitions in network types

Transitions in Social Network Types
$[7,8,25]$. Using recent national longitudinal data on older adults from Singapore, we examined social network types, stability, and change in network types over time and whether baseline and change in health and baseline sociodemographic characteristics were associated with network transitions.

We identified 5 types of social networks - diverse, unmarried and diverse, extended family, immediate family, and restricted among older Singaporeans. This differed from the 4 social network types (diverse, family, friend, and restricted) commonly reported in the literature (e.g., $[7,8]$ ). Specifically, as hypothesized (hypothesis 1), about one-fifth of older Singaporeans were categorized into the extended family type. Older adults in this type were highly likely to be married, have living children, live together with others, have 3 or more relatives to contact at least once a month, and have frequent contact with them. The importance of extended family networks can be pronounced in collectivistic Asian societies where kinshipbased support exchanges are prevalent and pivotal [12]. Specifically, in addition to the geographical proximity of older adults to their direct and extended family members in a small city-state, family-based networks have been promoted by policy initiatives in Singapore to nurture intergenerational support exchanges [42]. For instance, preferential terms for loans and placement for government-built housing are available for families that choose to live with or near their family members [43]. We thus expect the continued prevalence of extended familybased networks among older Singaporeans.

A substantial proportion of older Singaporeans belonged to the unmarried and diverse social network type, a type unique to our study. Older adults in this type maintained a diversified social network despite the absence of spousal ties and a higher likelihood of living alone than those in the other types. In particular, 67\% (297 out of 448 ) of unmarried respondents and $60 \%$ (84 out of 141) of solo-dwelling respondents at wave 2 were classified into the unmarried and diverse type. Although being unmarried or solitary living has been deemed a signal of social disconnectedness, our findings showed that they do not necessarily mean aging alone in isolation [44]. This is in line with a recent study reporting that about $14 \%$ of older Europeans who lived alone maintained diverse social networks [45]. We expect that future typology studies will also observe interesting variations in older adults' social network types, such as the unmarried and diverse type, in contemporary aging societies.

Around half of older Singaporeans' social network types changed in 2 years, with a slightly higher proportion 
expanding their networks than shrinking them. Therefore, our second hypothesis, which postulated network contraction over time, was not supported. Instead, the findings question the literature that suggests social withdrawal to be the norm in later life [14], while highlighting the dynamic nature of social networks [13] even over a relatively short time span. We also revealed that older adults did not only maintain or add ties with their close family but they were also able to expand or retain their networks beyond their personal boundaries. For instance, of 49 respondents newly widowed between waves 1 and 2, 24\% transitioned into more diverse types (from immediate/extended family types to the unmarried and diverse type), and $47 \%$ retained their network diversity (from diverse to unmarried and diverse types). This indicates that some older adults were proactive and resilient enough to maintain or cultivate diverse networks when they lost their spouse $[13,45]$. The findings also correspond to Litwin and Levinsky [16], who showed that more than onethird of older Europeans, who had no network in 2011, gained one or more confidant ties outside their close family (children and spouse) in 2015.

We found that (1) baseline depressive symptoms and increasing levels of depressive symptoms; (2) increased number of functional difficulties; and (3) age, gender, and education were associated with a transition into less diverse network types. Therefore, our third hypothesis, positing the association of declining health and low SES with social network contraction, was supported. Depressive symptoms are closely related to distorted self-perception (e.g., negative view of self) or perceived isolation (e.g., feelings of loneliness or lack of perceived support), which in turn leads to social disconnectedness [46, 47]. Moreover, older adults with worsening depressive symptoms may underrate, misinterpret, or find it difficult to reciprocate social support provided by social ties outside their kin, resulting in losing weak ties in their social networks over time [7]. We also observed the importance of functional health in social network composition, thereby siding with a study that reported a significant association between increased functional difficulties and declines in social network size [3]. Overall, we suggest that deterioration in mental and functional health contributes to loss of diversity in older adults' social networks [18], although it is not our intention to establish a causal direction between health and network changes using measures derived from both waves.

Additionally, the positive association between older age and network contraction to some extent validated theories, proposing a reduced network diversity and net- work concentration as people age $[14,15]$. Concerning gender differences, females were more likely to retain their social network than experience network expansion or contraction. This is due in part to the selection effect of females on network diversity: females tended to be classified into more diverse network types at baseline and maintained their network diversity over time. For instance, online supplementary Table 4 shows that females were more likely to be categorized into the unmarried and diverse type and less likely to be categorized into the immediate family type at baseline, compared to the extended family type. Indeed, $80 \%$ of those in the unmarried and diverse type were females (Table 2), and $86 \%$ of older adults in this type retained their type at wave 2 (Table 3 ). These findings are on par with past studies that have reported the gendered characteristics of social network composition: women tend to enjoy more diversified support networks than men throughout their life course [4, 19]. Last, highly educated older adults were less likely to experience network contraction. This reaffirms that education provides opportunities, resources, and skills for the maintenance of diverse social networks in later life $[19,21]$.

This study has some limitations. First, the availability of only 2 waves constrained the identification of transition trajectories. A recent study tracking older Americans for 10 years found homeostasis in older adults' social networks due to balancing between gains and losses in social ties over the life course [13]. Future studies need to explore patterns of transitions in social network types for a longer time span, assessing network indicators at frequent intervals. The impact of network transitions on subsequent physical and mental health can also be examined with additional data. As shown in a recent study, a reciprocal association between social network types and depressive symptoms is plausible [48]. Second, the potential bias subsequent to reduction in sample size due to random allocation of the questionnaire version and/or attrition, which could not be fully captured by MIs in supplementary analyses, might affect our findings. Third, the social network indicators used in this study, the number and frequency of contact with friends/relatives, mainly focused on the subjective structure of social networks (e.g., 6). These indicators may not reflect actual social ties as accurately as those derived from name generators [10]. Furthermore, the quality and function of social networks can be further considered in explicating social network types and their transitions in future studies [49].

These limitations notwithstanding, this study contributes to the current understanding of the literature on so- 
cial network typology by examining transitions in social network types in a rapidly aging Asian society using LTA. We suggest more studies should examine dynamics in social network types in other parts of the world. Practically, identifying older adults' existing social network types and their transitions can help researchers and practitioners to have increased awareness of older adults' changing social network composition over time. Policymakers should pay attention to older adults who remain in or transition into the restricted social network type over time to counteract risks arising from social disconnectedness. Additionally, public health initiatives should orchestrate tailored interventions for older adults with low SES and worsening functional and mental health to maintain diversity in their social networks.

\section{Statement of Ethics}

The study received approval from the Institutional Review Board at National University of Singapore, approval number B-15152. Written informed consent was obtained from participants of THE SIGNS Study waves 1 and 2, prior to the survey administration.

\section{Conflict of Interest Statement}

The authors have no conflicts of interest to declare.

\section{Funding Sources}

THE SIGNS study waves I and II were supported by Singapore's Ministry of Health $(\mathrm{MOH})$ under the agreement number MOH-NUS RL2015-053.

\section{Author Contributions}

Pildoo Sung planned the study, performed all data analysis, and drafted the paper. Rahul Malhotra and Grand H.-L. Cheng contributed to planning the study, data analysis and interpretation, and revising the paper. Angelique Chan contributed to planning the study and revising the paper.

\section{Data Availability Statement}

The complete THE SIGNS study dataset is not publicly available. The data used this study is available upon reasonable request. Please contact the corresponding author Pildoo Sung (pildoo. sung@duke-nus.edu.sg) for further information.

\section{References}

1 Moren-Cross JL, Lin N. Social networks and health. In: Binstock RH, George LK, Cutler SJ, Hendricks J, Schulz JH, editors. Handbook of aging and the social sciences. 6th ed. Burlington: Academic Press; 2006. p. 111-26.

2 Cornwell B, Laumann EO, Schumm LP. The social connectedness of older adults: a national profile. Am Sociol Rev. 2008;73(2):185203.

3 Cornwell B, Laumann EO. The health benefits of network growth: new evidence from a national survey of older adults. Soc Sci Med. 2015; 125:94-106.

4 Schwartz E, Litwin H. Social network changes among older Europeans: the role of gender. Eur J Ageing. 2018;15(4):359-67.

5 Litwin H. Social network type and morale in old age. Gerontologist. 2001;41(4):516-24.

6 Park NS, Jang Y, Lee BS, Chiriboga DA, Chang S, Kim SY. Associations of a social network typology with physical and mental health risks among older adults in South Korea. Aging Ment Health. 2018;22(5):631-8.

$7 \mathrm{Li} \mathrm{T}$, Zhang Y. Social network types and the health of older adults: exploring reciprocal associations. Soc Sci Med. 2015;130:59-68.

8 Kim B, Park S, Antonucci TC. Longitudinal changes in social networks, health and wellbeing among older Koreans. Ageing Soc. 2016; 36(9):1915-36

Transitions in Social Network Types
9 Fiori KL, Antonucci TC, Akiyama H. Profiles of social relations among older adults: a crosscultural approach. Ageing Soc. 2008;28(2): 203-31.

10 Litwin H, Stoeckel KJ. Confidant network types and well-being among older Europeans. Gerontologist. 2014;54(5):762-72.

11 Litwin H, Shiovitz-Ezra S. Social network type and subjective well-being in a national sample of older Americans. Gerontologist. 2011; 51(3):379-88.

12 Cheng ST, Lee CK, Chan AC, Leung EM, Lee JJ. Social network types and subjective wellbeing in Chinese older adults. J Gerontol B Psychol Sci Soc Sci. 2009;64(6):713-22.

13 Cornwell B, Goldman A, Laumann EO. Homeostasis revisited: patterns of stability and rebalancing in older adults' social lives. J Gerontol B Psychol Sci Soc Sci. 2021 Mar 14; 76(4):778-89.

14 Carstensen LL. The influence of a sense of time on human development. Science. 2006; 312(5782):1913-5.

15 Antonucci TC, Ajrouch KJ, Birditt KS. The convoy model: explaining social relations from a multidisciplinary perspective. Gerontologist. 2014;54(1):82-92.

16 Litwin H, Levinsky M. Always alone? Network transitions among detached older Europeans and their effects. Ageing Soc. 2020; 41(10):1-15.
17 Marsden PV. Network data and measurement. Annu Rev Sociol. 2003;16(1):435-63.

18 Schafer MH. Structural advantages of good health in old age: investigating the health-begets-position hypothesis with a full social network. Res Aging. 2013;35(3):348-70.

19 Ajrouch KJ, Blandon AY, Antonucci TC. Social networks among men and women: the effects of age and socioeconomic status. J Gerontol B Psychol Sci Soc Sci. 2005;60(6):S311-7.

20 Cornwell B, Schumm LP, Laumann EO, Graber J. Social networks in the NSHAP study: rationale, measurement, and preliminary findings. J Gerontol B Psychol Sci Soc Sci. 2009;64(Suppl 1):i47-55.

21 Van Groenou MIB, Van Tilburg T. Network size and support in old age: differentials by socio-economic status in childhood and adulthood. Ageing Soc. 2003;23(5):625-45.

22 Malhotra R, Bautista MAC, Müller AM, Aw $\mathrm{S}$, Koh GCH, Theng Y-L, et al. The aging of a young nation: population aging in Singapore. Gerontologist. 2019;59(3):401-10.

23 Nylund KL. Latent transition analysis: modeling extensions and an application to peer victimization [Doctoral dissertation]. Los Angeles: University of California; 2007.

24 Collins LM, Lanza ST. Latent class and latent transition analysis: with applications in the social, behavioral, and health sciences. Hoboken, New Jersey: John Wiley \& Sons; 2010. 
25 Litwin H, Levinsky M, Schwartz E. Network type, transition patterns and well-being among older Europeans. Eur J Ageing. 2020; 17(2):241-50

26 Cheng GH-L, Chan A, Østbye T, Malhotra R. Productive engagement patterns and their association with depressive symptomatology, loneliness, and cognitive function among older adults. Aging Ment Health. 2021;25(2): $332-40$.

27 Chan A, Saito Y, Matchar DB, Østbye T, Malhotra C, Ang S, et al. Cohort profile: panel on health and ageing of Singaporean elderly (PHASE). Int J Epidemiol. 2019;48(6):17501f.

28 White IR, Royston P, Wood AM. Multiple imputation using chained equations: issues and guidance for practice. Stat Med. 2011; 30(4):377-99.

29 Rinne LF, Ye A, Jordan NC. Development of fraction comparison strategies: a latent transition analysis. Dev Psychol. 2017;53(4):71330.

30 Ryoo JH, Wang C, Swearer SM, Hull M, Shi D. Longitudinal model building using latent transition analysis: an example using school bullying data. Front Psychol. 2018;9:675.

31 Morrow-Howell N, Putnam M, Lee YS, Greenfield JC, Inoue M, Chen $\mathrm{H}$. An investigation of activity profiles of older adults. $]$ Gerontol B Psychol Sci Soc Sci. 2014;69(5): $809-21$.

32 Lubben J, Blozik E, Gillmann G, Iliffe S, von Renteln Kruse W, Beck JC, et al. Performance of an abbreviated version of the Lubben social network scale among three European community-dwelling older adult populations. Gerontologist. 2006;46(4):503-13.
33 MacCallum RC, Zhang S, Preacher KJ, Rucker DD. On the practice of dichotomization of quantitative variables. Psychol Methods. 2002;7(1):19-40.

34 Li S, Stampfer MJ, Williams DR, VanderWeele TJ. Association of religious service attendance with mortality among women. JAMA Intern Med. 2016;176(6):777-85.

35 Kohout FJ, Berkman LF, Evans DA, CornoniHuntley J. Two shorter forms of the CES-D (Center for Epidemiological Studies Depression) depression symptoms index. J Aging Health. 1993;5(2):179-93.

36 Muthén B, Asparouhov T. LTA in Mplus: transition probabilities influenced by covariates. Mplus Web Notes. 2011;13:1-30.

37 Asparouhov T, Muthén B. Auxiliary variables in mixture modeling: three-step approaches using Mplus. Struct Equ Model. 2014;21(3): 329-41.

38 Lo Y, Mendell NR, Rubin DB. Testing the number of components in a normal mixture. Biometrika. 2001;88(3):767-78.

39 Satorra A, Bentler PM. A scaled difference chi-square test statistic for moment structure analysis. Psychometrika. 2001;66(4):507-14.

40 Pavlov G, Shi D, Maydeu-Olivares A. Chisquare difference tests for comparing nested models: an evaluation with non-normal data. Struct Equ Model. 2020;27(6):908-17.

41 Moore SA, Dowdy E, Nylund-Gibson K, Furlong MJ. A latent transition analysis of the longitudinal stability of dual-factor mental health in adolescence. J Sch Psychol. 2019;73: 56-73.
42 Thang LL. Social networks and the wellbeing of older adults in Singapore. In: Cheng S-T, Chi I, Fung HH, Li LW, Woo J, editors. Successful aging: Asian perspectives. Dordrecht: Springer Netherlands; 2015. p. 147-63.

43 Chan A, Matchar DB. Demographic and structural determinants of successful aging in Singapore. In: Cheng S-T, Chi I, Fung HH, Li LW, Woo J, editors. Successful aging: Asian perspectives. Dordrecht: Springer Netherlands; 2015. p. 65-79.

44 Carr D. Aging alone? International perspectives on social integration and isolation. J Gerontol B Psychol Sci Soc Sci. 2019;74(8): 1391-3.

45 Djundeva M, Dykstra PA, Fokkema T. Is living alone "aging alone"? Solitary living, network types, and well-being. J Gerontol B Psychol Sci Soc Sci. 2019;74(8):1406-15.

46 Amann G. Social network and social support deficits in depressed patients: a result of distorted perception? Eur Arch Psychiatry Clin Neurosci. 1991;241(1):49-56.

47 Santini ZI, Jose PE, York Cornwell E, Koyanagi A, Nielsen L, Hinrichsen C, et al. Social disconnectedness, perceived isolation, and symptoms of depression and anxiety among older Americans (NSHAP): a longitudinal mediation analysis. Lancet Public Health. 2020;5(1):e62-70.

48 Schwartz E, Litwin $\mathrm{H}$. The reciprocal relationship between social connectedness and mental health among older European adults: a SHARE-based analysis. J Gerontol B Psychol Sci Soc Sci. 2019;74(4):694-702.

49 Fiori KL, Antonucci TC, Cortina KS. Social network typologies and mental health among older adults. J Gerontol B Psychol Sci Soc Sci. 2006;61(1):P25-32. 